\title{
Lymphatic Vessel Neoplasm
}

National Cancer Institute

\section{Source}

National Cancer Institute. Lymphatic Vessel Neoplasm. NCI Thesaurus. Code C3723.

A benign or malignant neoplasm arising from the lymphatic vessels. 